\title{
Correction to: The role of physical activity in metabolic homeostasis before and after the onset of type 2 diabetes: an IMI DIRECT study
}

\author{
Robert W. Koivula ${ }^{1,2}$ (1) $\cdot$ Naeimeh Atabaki-Pasdar ${ }^{1}$ - Giuseppe N. Giordano ${ }^{1} \cdot$ Tom White $^{3} \cdot$ Jerzy Adamski $^{4,5,6}$. \\ Jimmy D. Bell ${ }^{7}$. Joline Beulens ${ }^{8}$. Søren Brage ${ }^{3,9}$. Søren Brunak ${ }^{10,11}$ - Federico De Masi ${ }^{10,11}$. \\ Emmanouil T. Dermitzakis ${ }^{12,13,14} \cdot$ Ian M. Forgie ${ }^{15} \cdot$ Gary Frost $^{16} \cdot$ Torben Hansen $^{9,17} \cdot$ Tue H. Hansen $^{17}$. \\ Andrew Hattersley ${ }^{18,19} \cdot$ Tarja Kokkola $^{20} \cdot$ Azra Kurbasic $^{1} \cdot$ Markku Laakso $^{20} \cdot$ Andrea Mari $^{21} \cdot$ Timothy J. McDonald $^{18}$. \\ Oluf Pedersen ${ }^{17} \cdot$ Femke Rutters $^{8} \cdot$ Jochen M. Schwenk $^{22}$ • Harriet J. A. Teare ${ }^{23} \cdot$ E. Louise Thomas ${ }^{7}$. \\ Ana Vinuela ${ }^{12,13,14}$. Anubha Mahajan ${ }^{24}$. Mark I. McCarthy ${ }^{2,24,25,26} \cdot$ Hartmut Ruetten $^{27}$ Mark Walker $^{28}$. \\ Ewan Pearson ${ }^{15} \cdot$ Imre Pavo $^{29} \cdot$ Paul W. Franks ${ }^{1,2,30,31} \cdot$ for the IMI DIRECT Consortium
}

Published online: 13 November 2020

(C) Springer-Verlag GmbH Germany, part of Springer Nature 2020

\section{Correction to: Diabetologia}

https://doi.org/10.1007/s00125-019-05083-6

Unfortunately, 'Present address' was omitted from one of the addresses provided for Mark I. McCarthy (\#26). The corrected address details are given on the following page.

Publisher's note Springer Nature remains neutral with regard to jurisdictional claims in published maps and institutional affiliations.

The online version of the original article can be found at https://doi.org/ 10.1007/s00125-019-05083-6

Robert W. Koivula

robert.koivula@med.lu.se

Extended author information available on the last page of the article 


\section{Affiliations}

Robert W. Koivula ${ }^{1,2}$ (D) Naeimeh Atabaki-Pasdar ${ }^{1}$. Giuseppe N. Giordano ${ }^{1} \cdot$ Tom White $^{3}$. Jerzy Adamski ${ }^{4,5,6}$. Jimmy D. Bell ${ }^{7}$. Joline Beulens ${ }^{8}$. Søren Brage ${ }^{3,9}$ - Søren Brunak ${ }^{10,11} \cdot$ Federico De Masi $^{10,11}$. Emmanouil T. Dermitzakis ${ }^{12,13,14} \cdot$ Ian M. Forgie ${ }^{15} \cdot$ Gary Frost $^{16} \cdot$ Torben Hansen $^{9,17} \cdot$ Tue H. Hansen $^{17}$. Andrew Hattersley ${ }^{18,19}$ - Tarja Kokkola ${ }^{20}$ • Azra Kurbasic ${ }^{1} \cdot$ Markku Laakso $^{20} \cdot$ Andrea Mari $^{21} \cdot$ Timothy J. McDonald $^{18}$. Oluf Pedersen ${ }^{17}$. Femke Rutters ${ }^{8}$. Jochen M. Schwenk ${ }^{22}$ • Harriet J. A. Teare ${ }^{23}$ • E. Louise Thomas ${ }^{7}$.

Ana Vinuela ${ }^{12,13,14}$. Anubha Mahajan ${ }^{24}$. Mark I. McCarthy ${ }^{2,24,25,26} \cdot$ Hartmut Ruetten $^{27}$ - Mark Walker ${ }^{28}$. Ewan Pearson ${ }^{15} \cdot$ Imre Pavo $^{29} \cdot$ Paul W. Franks ${ }^{1,2,30,31} \cdot$ for the IMI DIRECT Consortium

1 Department of Clinical Sciences, Lund University, Genetic and Molecular Epidemiology, CRC, Skåne University Hospital Malmö, Building 91, Level 12, Jan Waldenströms gata 35, SE-205 02 Malmö, Sweden

2 Oxford Centre for Diabetes, Endocrinology and Metabolism, Radcliffe Department of Medicine, University of Oxford, Oxford, UK

3 MRC Epidemiology Unit, University of Cambridge School of Clinical Medicine, Cambridge, UK

4 Research Unit Molecular Endocrinology and Metabolism, Genome Analysis Center, Helmholtz Zentrum München, German Research Center for Environmental Health, Neuherberg, Germany

5 Lehrstuhl für Experimentelle Genetik, Technische Universität München, Freising-Weihenstephan, Germany

6 Department of Biochemistry, Yong Loo Lin School of Medicine, National University of Singapore, Singapore, Republic of Singapore

7 Research Centre for Optimal Health, Department of Life Sciences, University of Westminister, London, UK

8 Department of Epidemiology and Biostatistics, Amsterdam Public Health Research Institute, Amsterdam University Medical Centre, Location VU University Medical Center, Amsterdam, the Netherlands

9 Faculty of Health Sciences, University of Southern Denmark, Odense, Denmark

10 The Novo Nordisk Foundation Center for Protein Research, University of Copenhagen, Copenhagen, Denmark

11 Department of Bio and Health Informatics, Technical University of Denmark, Lyngby, Denmark

12 Department of Genetic Medicine and Development, University of Geneva Medical School, Geneva, Switzerland

13 Institute of Genetics and Genomics in Geneva (iGE3), University of Geneva, Geneva, Switzerland

14 Swiss Institute of Bioinformatics, Geneva, Switzerland

15 Population Health \& Genomics, School of Medicine, University of Dundee, Ninewells Hospital, Dundee, UK
16 Nutrition and Dietetics Research Group, Department of Medicine, Division of Diabetes, Endocrinology and Metabolism, Imperial College London, Hammersmith Campus, London, UK

17 The Novo Nordisk Foundation Center for Basic Metabolic Research, Faculty of Health and Medical Science, University of Copenhagen, Copenhagen, Denmark

18 NIHR Exeter Clinical Research Facility, University of Exeter Medical School, Exeter, UK

19 Institute of Biomedical and Clinical Science, University of Exeter Medical School, Exeter, UK

20 Department of Medicine, University of Eastern Finland and Kuopio University Hospital, Kuopio, Finland

21 Institute of Neurosciences, National Research Council, Padova, Italy

22 Affinity Proteomics, Science for Life Laboratory, KTH - Royal Institute of Technology, Stockholm, Sweden

23 HeLEX, Nuffield Department of Population Health, University of Oxford, Old Road Campus, Headington, Oxford, UK

24 Wellcome Centre for Human Genetics, University of Oxford, Oxford, UK

25 NIHR Oxford Biomedical Research Centre, Churchill Hospital, Oxford, UK

26 Present address: Human Genetics, Genentech, South San Francisco, CA, USA

27 Sanofi-Aventis Deutschland GmbH, R\&D, Frankfurt am Main, Germany

28 Institute of Cellular Medicine (Diabetes), Newcastle University, Newcastle upon Tyne, UK

29 Eli Lilly Regional Operations GmbH, Vienna, Austria

30 Department of Nutrition, Harvard School of Public Health, Boston, MA, USA

31 Department of Public Health \& Clinical Medicine, Section for Medicine, Umeå University Hospital, Umeå, Sweden 